\title{
SOLVABLE ASSOSYMMETRIC RINGS ARE NILPOTENT
}

\author{
DAVID POKRASS AND DAVID RODABAUGH
}

\begin{abstract}
Assosymmetric rings are ones which satisfy the law $(x, y, z)$ $=(P(x), P(y), P(z))$ for each permutation $P$ of $x, y, z$. Let $A$ be an assosymmetric ring having characteristic different from 2 or 3 . We show that if $A$ is solvable then $A$ is nilpotent. Also, if each subring generated by a single element is nilpotent, and if $A$ has D.C.C. on right ideals, then $A$ is nilpotent. We also give an example showing that the Wedderburn Principal Theorem fails for assosymmetirc rings.
\end{abstract}

1. Introduction. A nonassociative ring is called nilpotent if there is a fixed positive integer $t$ such that any product involving $t$ elements is 0 . A ring is solvable if the chain of subrings $A \supseteq A^{2} \supseteq\left(A^{2}\right)^{2} \supseteq \cdots$ reaches 0 in a finite number of steps. While solvable associative rings are obviously nilpotent, solvable alternative rings need not be [1]. An assosymmetric ring is one which satisfies the condition $(x, y, z)=(P(x), P(y), P(z))$ for each permutation $P$ of $x, y, z$. These rings were introduced by Kleinfeld [2]. He showed that an assosymmetric ring having characteristic different from 2 and 3 was either associative or it had a nonzero ideal whose square was zero.

From here on $A$ will denote an assosymmetric ring having characteristic different from 2 and 3 . The main purpose of this note is to show that $A$ is solvable if and only if $A$ is nilpotent. We will let $A^{*}$ denote the ring generated by the right and left multiplication operators $R_{x}$ and $L_{x}, x \in A$. Furthermore we will say that $A$ is right nilpotent (of index $n$ ) if for some fixed $n, R_{x_{1}} \cdots R_{x_{n}}$ $=0$ for all $x_{i}$. Similarly we may define $A$ to be left nilpotent. It is not difficult to show that all nilpotent rings are right nilpotent and that all right nilpotent rings are solvable.

The following identities in $A^{*}$ will be useful:

$$
\begin{gathered}
R_{y} L_{x}=L_{x} R_{y}-R_{y} R_{x}+R_{y x}, \\
L_{y} L_{x}=L_{x y}-R_{x} R_{y}+R_{x y}, \\
R_{x} R_{y}=R_{x y}-R_{y x}+R_{y} R_{x}, \\
0=R_{y} R_{z} R_{w} R_{x}-R_{y z} R_{w} R_{x}-R_{y} R_{z} R_{w x}+R_{y z} R_{w x} .
\end{gathered}
$$

Received by the editors July 14, 1976 and, in revised form, October 8, 1976. AMS (MOS) subject classifications (1970). Primary 17A30. 
Identities (1) and (2) are both equivalent to the law $(a, b, c)=(c, a, b)$. Identity (3) is equivalent to $(a, b, c)=(a, c, b)$. Identity (4) is a restatement of equation 11 in [2], which says that $0=((t, y, z), w, x)$.

\section{Nilpotent rings.}

LEMMA 1. Every product $S_{x_{1}} S_{x_{2}} \cdots S_{x_{k}}$ in $A^{*}$ may be rewritten as a sum of terms of the form $L L \cdots L R R \cdots R$ where the number of $R$ 's appearing in each term is at least as great as the number of $R$ 's in $S_{x_{1}} S_{x_{2}} \cdots S_{x_{k}}$.

Proof. Let $T=S_{x_{1}} S_{x_{2}} \cdots S_{x_{k}}$. We define $d\left(S_{x_{i}}\right)$ to be 0 if $S=R$, and if $S=L$ define $d\left(S_{x_{i}}\right)$ to be the number of $S$ 's preceding it. Finally, let $d(T)=\sum d\left(S_{x_{i}}\right)$, and we will call this the degree of $T$. We induct on $d(T)$. If $d(T)=0$ there is nothing to prove. Assume the lemma for each product of degree less than $m=d(T)>0$. We may assume that $T$ begins with an $R$, say

$$
T=R_{x_{1}} \cdots R_{x_{i-1}} L_{x_{i}} S_{x_{i+1}} \cdots S_{x_{k}}
$$

Using (1) this becomes

$$
\begin{aligned}
R_{x_{1}} \cdots L_{x_{i}} R_{x_{i-1}} S_{x_{i+1}} \cdots S_{x_{k}}-R_{x_{1}} \cdots R_{x_{i-1}} & R_{x_{i}} S_{x_{i+1}} \cdots S_{x_{k}} \\
& +R_{x_{1}} \cdots R_{x_{i-1} x_{i}} S_{x_{i+1}} \cdots S_{x_{k}}
\end{aligned}
$$

a sum of three terms, each of degree less than $m$, and each having at least as many $R$ 's as $T$ had. By induction we are done. From Lemma 1 and a symmetric argument we get

LEMMA 2. If $A$ is right nilpotent of index $n$ then any product involving at least $n R$ 's is zero. If $A$ is left nilpotent of index $m$ then any product involving $m$ L's is zero.

LEMMA 3. If $A$ is both left and right nilpotent then $A$ is nilpotent.

Proof. Assume the index of right nilpotence is $n$ and the index of left nilpotence is $m$. To show $A$ is nilpotent it is sufficient to show $A^{*}$ is nilpotent [4]. However any product of $n+m$ elements in $A^{*}$ is a sum of terms each involving at least $n R$ 's or $m L$ 's. By Lemma 2 each term is 0 .

LEMMA 4. If $A$ is right nilpotent then $A$ is nilpotent.

Proof. By Lemma 3 we need only show that $A$ is also left nilpotent. Let us say the index of right nilpotence of $A$ is $n-1$, so that $\left(\left(x_{1} x_{2}\right) x_{3}\right) \cdots x_{n}=0$ for any $n$ elements in $A$. Consider equation (2):

$$
L_{x_{2}} L_{x_{1}}=L_{x_{1} x_{2}}-R_{x_{1}} R_{x_{2}}+R_{x_{1} x_{2}} .
$$

Left multiplication by $L_{x_{3}}$ shows that

$$
L_{x_{3}} L_{x_{2}} L_{x_{1}}=L_{x_{3}} L_{x_{1} x_{2}}-L_{x_{3}} R_{x_{1}} R_{x_{2}}+L_{x_{3}} R_{x_{1} x_{2}} .
$$


Using (2) again, now on the term $L_{x_{3}} L_{x_{1} x_{2}}$, gives

$$
L_{x_{3}} L_{x_{2}} L_{x_{1}}=L_{\left(x_{1} x_{2}\right) x_{3}}-R_{x_{1} x_{2}} R_{x_{3}}+R_{\left(x_{1} x_{2}\right) x_{3}}-L_{x_{3}} R_{x_{1}} R_{x_{2}}+L_{x_{3}} R_{x_{1} x_{2}} .
$$

Repeating, we multiply this last equation by $L_{x_{4}}$ and apply (2) to the term $L_{x_{4}} L_{\left(x_{1} x_{2}\right) x_{3}}$. Continuing, we arrive at

$$
L_{x_{n}} \cdots L_{x_{2}} L_{x_{1}}=L_{\left(\left(x_{1} x_{2}\right) \ldots\right) x_{n}}+\sum T_{i}
$$

where each $T_{i}$ is a term containing at least one $R$. Since $A$ is right nilpotent, $L_{x_{n}} \cdots L_{x_{2}} L_{x_{1}}=\sum T_{i}$. This shows that any product of $n^{2} L$ 's is a sum of terms each containing at least $n R$ 's. By right nilpotency and Lemma 2 each term is zero, and so $A$ is left nilpotent.

We remark that Lemma 4 actually holds for rings satisfying only the law $(a, b, c)=(c, a, b)$ since the only identities used thus far have been (1) and (2). Consequently, right nilpotent alternative rings are nilpotent. We now prove the main result of this section.

THEOREM 5. Let $A$ be a solvable assosymmetric ring of characteristic $\neq 2,3$. Then $A$ is nilpotent.

Proof. By Lemma 4 we need only show that $A$ is right nilpotent. Consider the ideal $B=A^{2}$. Since $B$ is solvable of lesser length than $A$ we may assume $B$ is nilpotent. Then we know $B^{*}$ is also nilpotent [4]. Next let us denote the subring in $A^{*}$ generated by $\left\{R_{x_{i}} \mid x_{i} \in A\right\}$ by $\hat{A}$. Identity (4) shows

$$
R_{y} R_{z} R_{w} R_{x} \equiv R_{y z} R_{w} R_{x} \bmod \hat{A} B^{*}
$$

Equation (3) shows

$$
R_{y z} R_{w} R_{x}=R_{(y z) w} R_{x}-R_{w(y z)} R_{x}+R_{w} R_{y z} R_{x} .
$$

Using (3) again on each of the three terms on the right-hand side of the last equation will give us $R_{y z} R_{w} R_{x} \in \hat{A} B^{*}+B^{*}$. This implies then that

$$
R_{y} R_{z} R_{w} R_{x} \in A B^{*}+B^{*}, \text { or }(\hat{A})^{4} \subseteq \hat{A} B^{*}+B^{*},
$$

so that $(\hat{A})^{5} \subseteq \hat{A} B^{*}$. Finally, an induction argument shows that $(\hat{A})^{4 i+1}$ $\subseteq \hat{A}\left(B^{*}\right)^{i}$ for each $i$. This means, since $B^{*}$ is nilpotent, that $\hat{A}$ is nilpotent, and hence, $A$ is right nilpotent.

Usually the concept of a nilring is reserved for power associative rings. However we shall define $A$ to be nil if each subring generated by a single element is nilpotent. Taking this as our definition we get

COROLlARY 6. Let $A$ be an assosymmetric nilring having characteristic $\neq 2,3$, and assume $A$ has the descending chain condition on right ideals. Then $A$ is nilpotent.

Proof. Let $J$ be the ideal generated by all associators $(a, b, c)$. It is shown in [2] that $J^{2}=0$. Since $A / J$ is an associative nilring with D.C.C. on right 
ideals, it is well known that $A / J$ is solvable. The solvability of $J$ and $A / J$ now guarantees that $A$ is solvable. By Theorem $5 A$ is nilpotent.

3. Nilpotent rings-A second approach. In the previous section certain results were proved primarily using the identity $(a, b, c)=(c, a, b)$. In this section we will generalize Theorem 5 . In [2] it was shown that if $A$ is an assosymmetric ring having characteristic different from 2 and 3 , then each associator $(a, b, c)$ was in the nucleus of $A$.

Lemma 7. Let $A$ be a ring and $S$ a subring. Assume $S^{k} \subseteq N$ for some $k \geqslant 1$, where $N$ is the nucleus of $A$. Then for each $m, n \geqslant 1$ there exists an $l \geqslant 1$ such that $S^{l} \subseteq\left(S^{m}\right)^{n}$.

Proof. We induct on $n$. For $n=1$ take $l=m$. Next assume $S^{l} \subseteq\left(S^{m}\right)^{n}$. Let $t=\max \{k, l\}$. We claim $S^{2 t+2 m-3}$ is contained in $\left(S^{m}\right)^{n+1}$. For choose $x \in S^{2 t+2 m-3}$. Then $x$ is a sum of terms each of the form $b=a_{1} a_{2} \cdots a_{s}$ where $s=2 t+2 m-3$ and each $a_{i} \in S$. If $u$ is a factor of $b$ in this particular association, define $d(u)$ as the number of the $a_{i}$ 's in $u$. (For example, if $b=a_{1}\left(\left(a_{2} a_{3}\right) a_{4}\right)$ then $d\left(a_{2} a_{3}\right)=2$. But in this association $a_{3} a_{4}$ is not even a factor.) Next, choose $y$ with $d(y) \geqslant t$ but otherwise $d(y)$ as small as possible. We claim $d(y) \leqslant 2 t-2$. For if $d(y) \geqslant 2 t-1$ then $y=u v$ in this association of the $a_{i}$ 's and $d(u)+d(v) \geqslant 2 t-1$. Thus $d(u) \geqslant t$ or $d(v) \geqslant t$, contrary to the choice of $y$. Since $S^{k} \subseteq N$ it is clear that $y \in N$ and the product of $y$ with any of the $a_{i}$ 's is also in $N$. Thus the $a_{i}$ 's can be reassociated so that $b=w y, y z$, or wyz under some association. If $b=w y z$ then

$$
d(w)+d(z) \geqslant(2 t+2 m-3)-(2 t-2)=2 m-1 .
$$

Therefore $d(w) \geqslant m$ or $d(z) \geqslant m$. We conclude that $b \in S^{m} S^{t} S^{r}, S^{r} S^{t} S^{n}$, $S^{m} S^{t}$, or $S^{t} S^{n}$. Hence $b \in\left(S^{m}\right)^{n+1}$, and $x \in\left(S^{m}\right)^{n+1}$.

The above lemma has some interesting consequences, some of which are not central to our purpose. However, we mention that, using Lemma 7, one can show that the sum of two locally nilpotent ideals is locally nilpotent provided one of them is contained in the nucleus.

We now prove the main result of this section.

THEOREM 8. Let $R$ be a ring having characteristic $\neq 2$ with each associator in the nucleus. Then if $R$ is solvable, $R$ is nilpotent.

Proof. In any ring $R, J=(R, R, R)+(R, R, R) R$ is an ideal. We first show that $J \subseteq N$. Using the familiar Teichmuiller identity and the fact that $(R, R, R) \subseteq N$, we get

$$
\begin{aligned}
(a, b, c)(x, y, z) & =(a, b, c(x, y, z))=-(a, b,(c, x, y) z \\
& =-(a, b(c, x, y), z)=(a,(b, c, x) y, z)=(a(b, c, x), y, z) \\
& =-((a, b, c) x, y, z)=-(a, b, c)(x, y, z) .
\end{aligned}
$$


By our characteristic assumption all of the above expressions become 0 . This shows $(R, R, R) R \subseteq N$ and so $J \subseteq N$. Now assume $R$ is solvable. Then $R / J$ is a solvable associative ring and therefore nilpotent. Hence $R^{k} \subseteq J \subseteq N$ for some $k$. Also $J$ is associative, so $J^{n}=0$. (With extra work one can actually show $J^{2}=0$.) We now apply Lemma 7 taking $S=R$ and $m=k$. Then there is an $l$ for which $R^{l} \subseteq\left(R^{k}\right)^{n} \subseteq J^{n}=0$. This shows $R$ is nilpotent and completes the proof.

COROLlaRY 9. Let $R$ be as in Theorem 8. If $R$ is nil with D.C.C. on right ideals then $R$ is nilpotent.

Proof. The proof of Corollary 6 will work if it is true that $J^{2}=0$. In the proof of Theorem 8 we showed $J \subseteq N$ and $(a, b, c)(x, y, z)=0$. These facts are enough to invoke the argument used in the main theorem of [2] in showing $J^{2}=0$.

4. An interesting counterexample. From the results above and in [2], one can see that the assosymmetric identities are powerful identities. In the presence of these theorems, one would expect the Wedderburn Principal Theorem to be proved in short order. In [3] the idempotent lifting theorem is proved under fairly general conditions. The problem with assosymmetric not associative rings is that they are not power associative.

Let $A$ be an algebra over $F$ spanned by $e, n$ with $e^{2}=e+n, n e=n$, en $=n^{2}=0$. Then $N=\{\alpha n \mid \alpha$ in $F\}$ is the radical of $A$ and $A / N$ is isomorphic to $F$. The ring $A$ is assosymmetric. However, if $e+\alpha n=(e+\alpha n)^{2}=e+n$ $+\alpha n$ then $\alpha+1=\alpha$. Thus, there are no nonzero idempotents in $A$. The Wedderburn Principal Theorem and the idempotent lifting theorem both fail for $A$. The fact that $A$ is only two dimensional would indicate that no meaningful results in these directions could be expected.

\section{REFERENCES}

1. G. V. Dorofeyev, An example of a solvable but nonnilpotent alternative ring, Uspehi Mat. Nauk 15 (1960), no. 3(93), 147-150; English transl., Amer. Math. Soc. Transl. (2) 37 (1964), $79-83$.

2. E. Kleinfeld, Assosymmetric rings, Proc. Amer. Math. Soc. 8 (1957), 983-986.

3. David Rodabaugh, On the Wedderburn principal theorem, Trans. Amer. Math. Soc. 138 (1969), 343-361.

4. R. Schafer, An introduction to non associative algebras, Pure and Appl. Math., vol. 22, Academic Press, New York, 1966.

Department of Mathematics, University of Missouri, Columbia, Missouri 65201 (Current address of David Rodabaugh)

Current address (David Pokrass): Department of Mathematics, Emory University, Atlanta, Georgia 30322 